\title{
Efficacy and Safety of Tazarotene 0.045\% Lotion in White Adults with Moderate-to-Severe Acne
}

James Q Del Rosso, DO'; Linda Stein Gold, MD²; Stephen K Tyring, MD, PhD³; William Philip Werschler, MD4; Fran E Cook-Bolden, MD5,6; George Han, MD, PhD; Neal Bhatia, MD; Eric Guenin, PharmD, PhD, MPH² "Therapeutics Clinical Research, San Diego, CA; 'Ortho Dermatologicst, Bridgewater, NJ
"Ortho Dermatologics is a division of Bausch Health US, LLC

SYNOPSIS

Acne is a common dermatologic condition that affects adults as well as adolescents It also manifests differently in different skin types, with a higher proportion of
noninflammatory acne (versus inflammatory) and moderate-to-severe erythema in

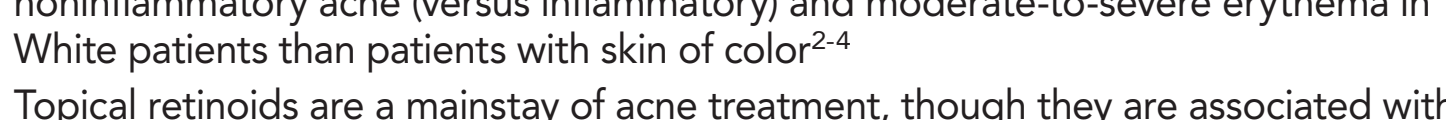
- Topical retinoids are a mainstay of acne treatric Tazarotene $0.045 \%$ polymeric emulsion lotion has demonstrated safety and efficacy in two pooled phase 3 stud
(Black, Asian, Hispanic)' OBJECTIVES

- To determine the efficacy and safety of tazarotene $0.045 \%$ lotion in White adults with
moderate-to-severe acce and evaluate its impact on quality of life METHODS

In two phase 3, double-blind, 12-week studies (NCT03168334; NCT03168321), - Cerava hydrating cleanser and Cerave ${ }^{\oplus}$ moisturizing lotion (L'Oreal, NY) were

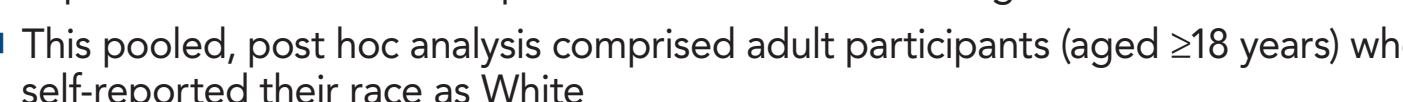

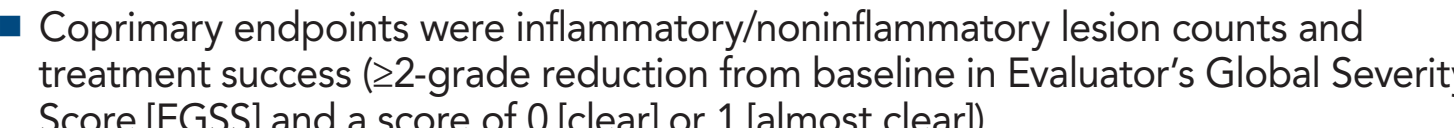

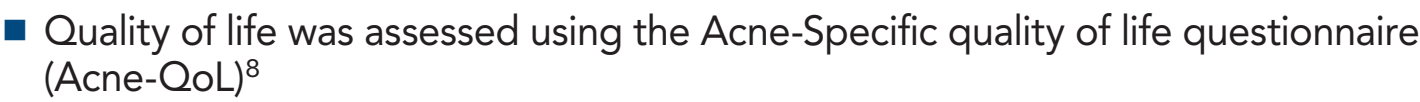
- Treatment-emergent adverse events (TEAEs) and cutaneous safety and tolerability
were also evaluated RESULTS

Demographics and Baseline Characteristics

Of 1614 participants in the intent to treat population (ITT) of the two pooled phase 3
studies, a total of 645 and 619 White adults comprised the ITT and safety populations

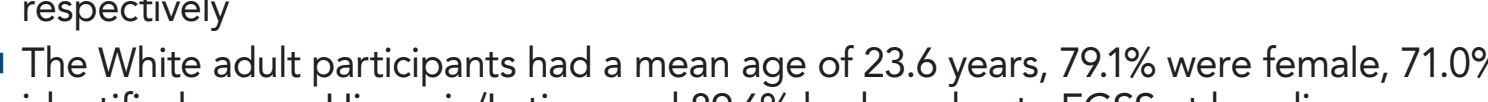
treatment groups except for a slightly greater percentage of females in the

fficacy and Quality of Life

- At week 12, tazarotene $0.045 \%$ lotion provided approximately $60 \%$ reductions in
inflammatory and noninflammatory lesion counts $(P<0.001$ vs vehicle, both; Figure 1$)$

- The percentage of participiants achieving treatment success and $E G S S$ improvements
at week 12 were significantly greater with tazarotene than vehicle $(P<0.001$ and $P<0.05$,

Quality of life was also improved at week 12, with greater numerical improvements in role-emotional, and role-social (Table 1)

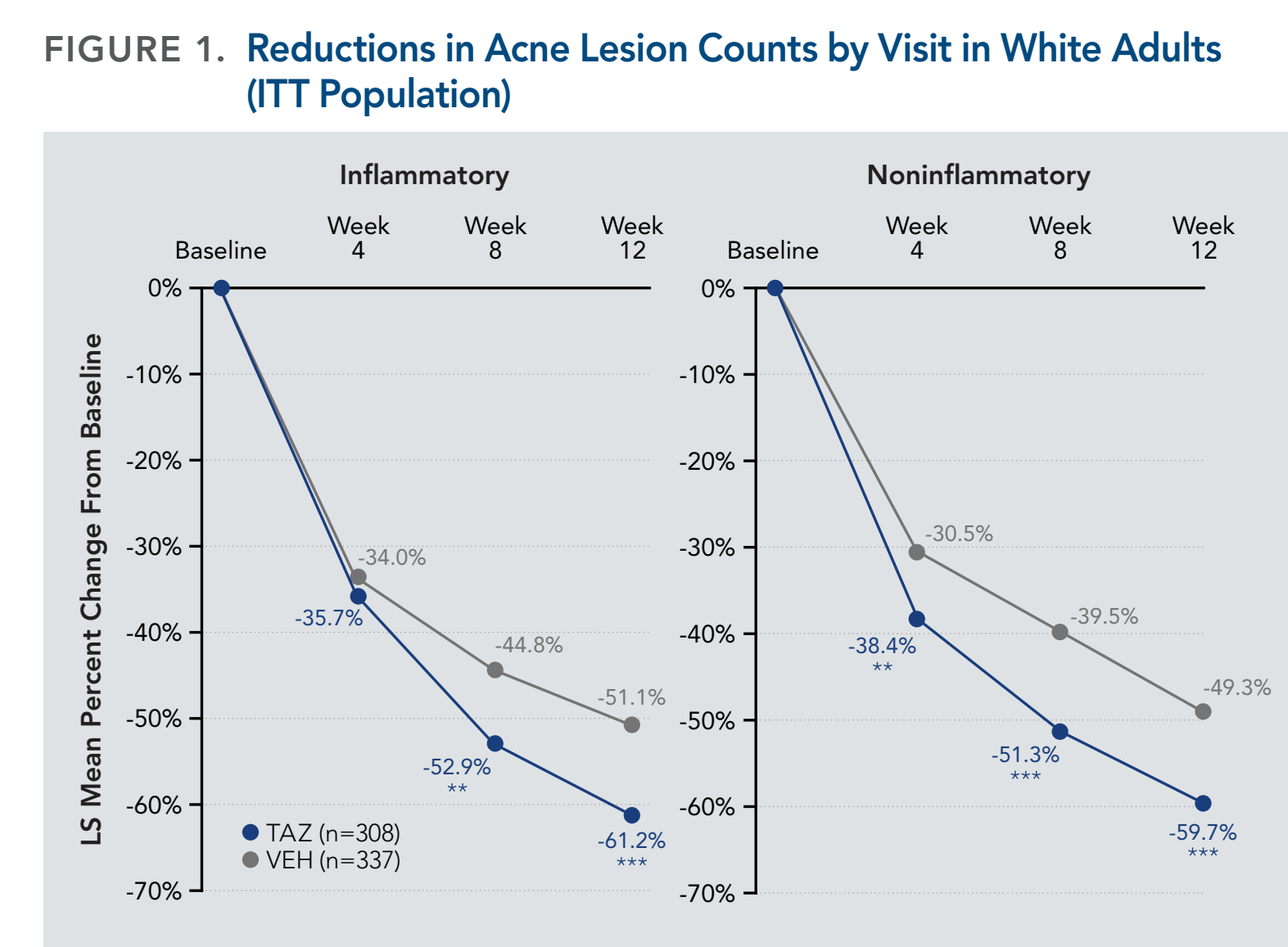

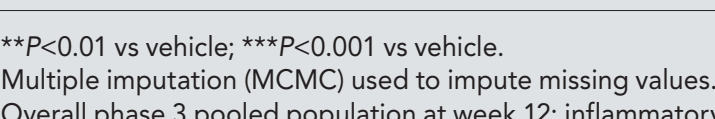$$
\text { .70\% }
$$

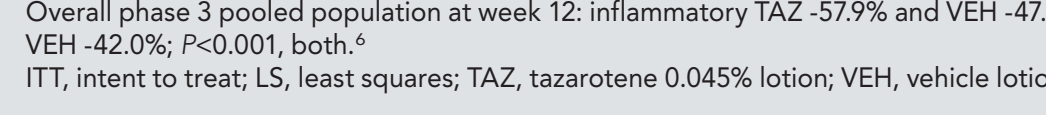

FIGURE 2. Achievement of Treatment Success ${ }^{a}$ or Mild/Almost Clear/
Clearb at Week 12 in White Adults (ITT Population)

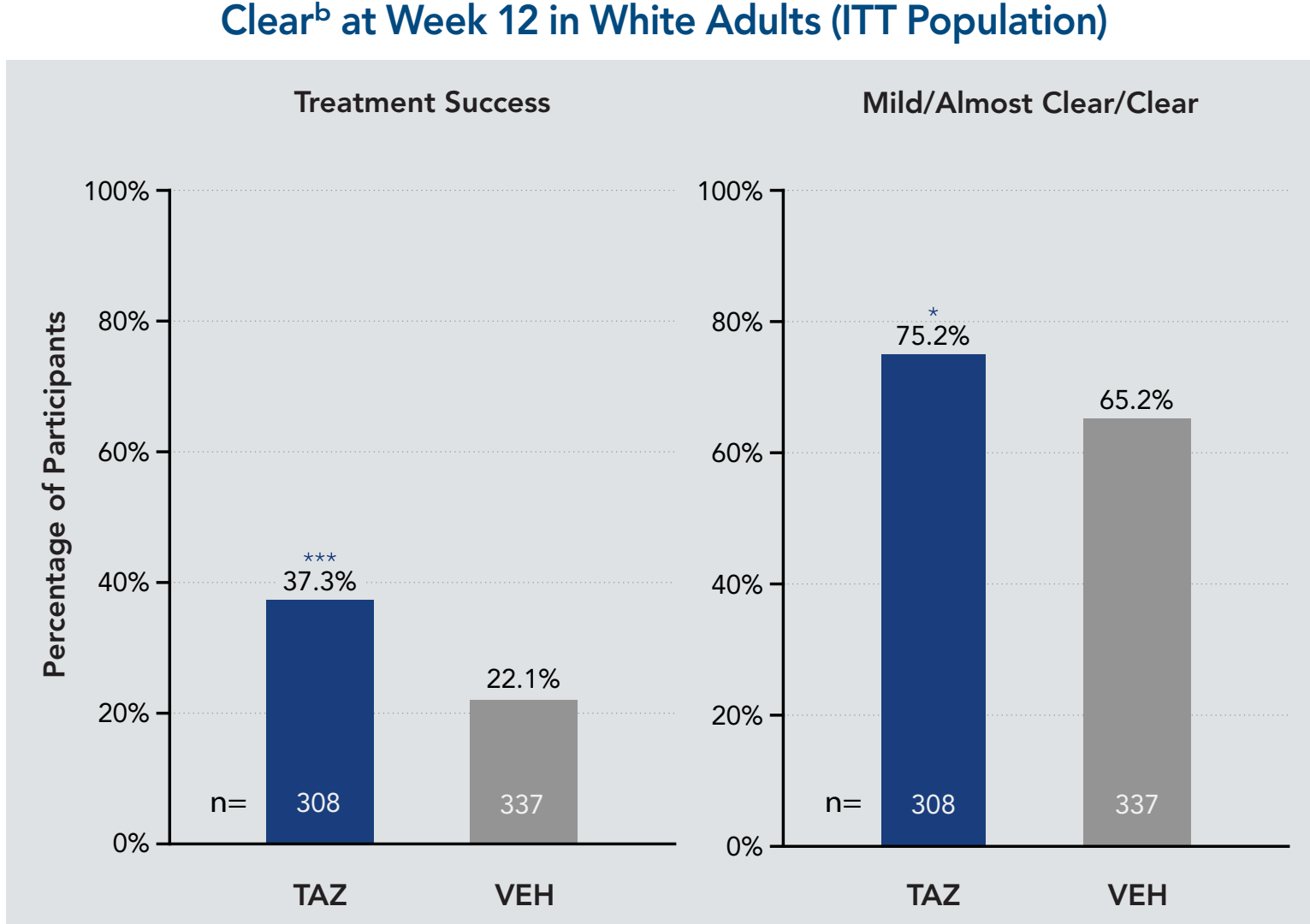

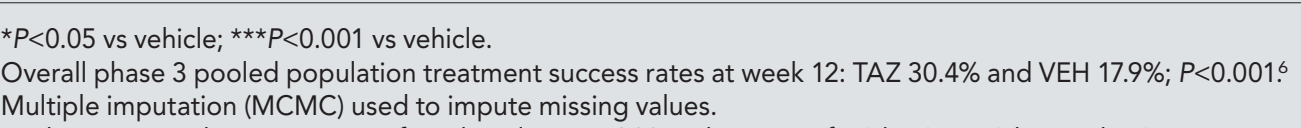

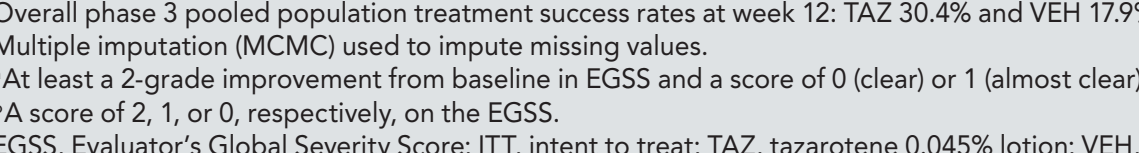

FIGURE 3. Acne Improvements With Tazarotene $0.045 \%$ Lotion

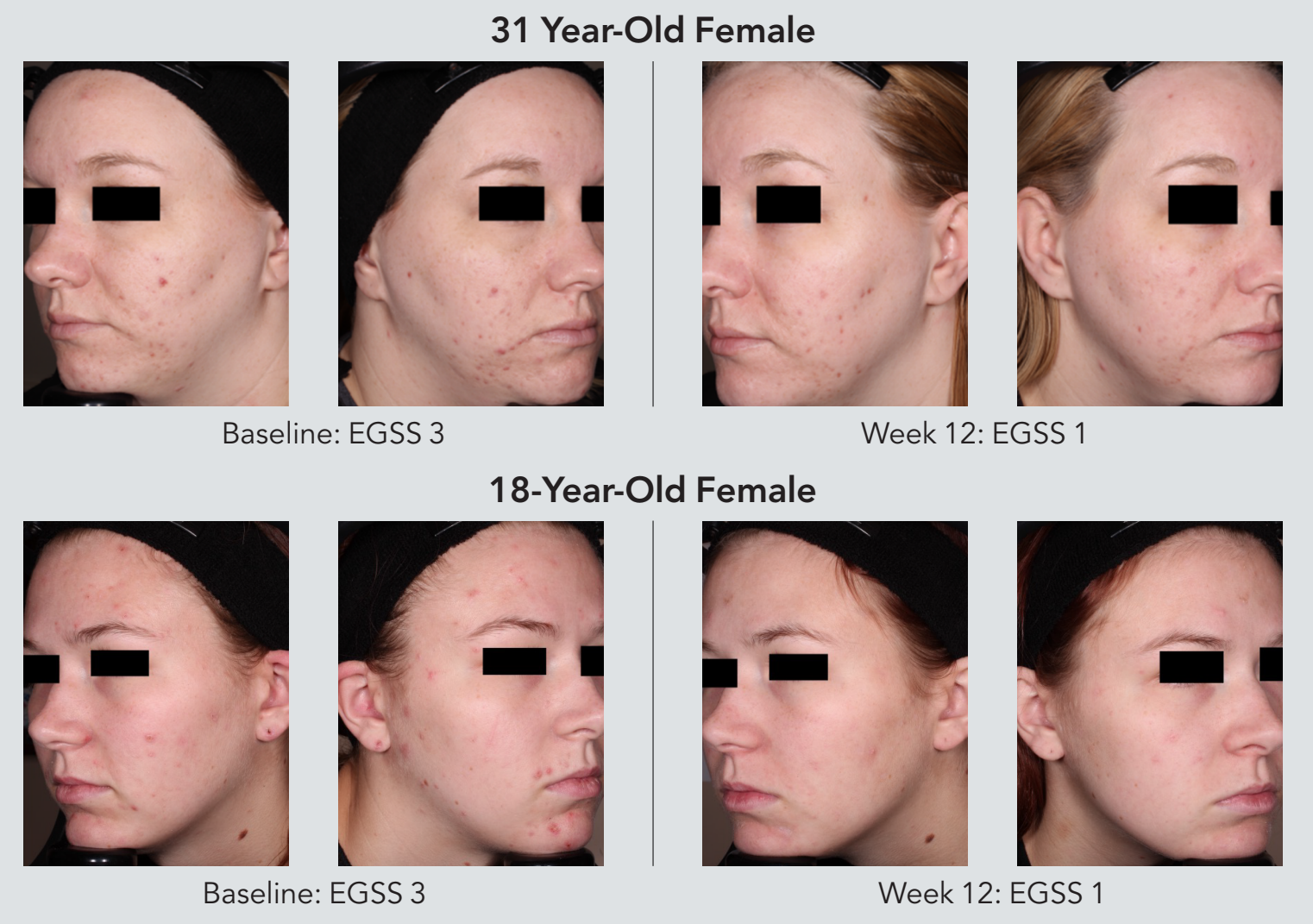

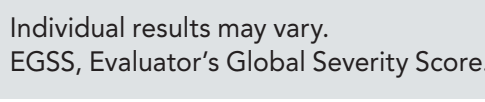

TABLE 1. Mean Change From Baseline in Acne-OoL Scores at Week 12 in White Adults (ITT Population)

\begin{tabular}{c|c|c}
\hline $\begin{array}{c}\text { Acne-OoL Domain Subgroup } \\
\text { mean (SD) change from baseline }\end{array}$ & $\begin{array}{c}\text { Tazarotene 0.045\% Lotion } \\
(n=250)\end{array}$ & $\begin{array}{c}\text { Vehicle Lotion } \\
(n=282)\end{array}$ \\
\hline
\end{tabular} \begin{tabular}{l|l|l|}
\hline Self-perception & $10.1(8.0)$ & $8.7(8.0)$ \\
\hline
\end{tabular} \begin{tabular}{l|l|l|}
\hline Role-emotional & $8.5(8.0)$ & $7.0(8.6)$ \\
\hline Role-social & $6.2(6.7)$ & $5.3(6.6)^{3}$ \\
\hline
\end{tabular} \begin{tabular}{l|c|c}
\hline Acne symptoms & $8.2(6.0)^{* *}$ & $6.5(6.2)$ \\
\hline
\end{tabular}

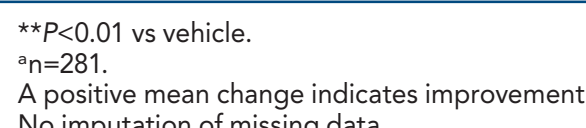

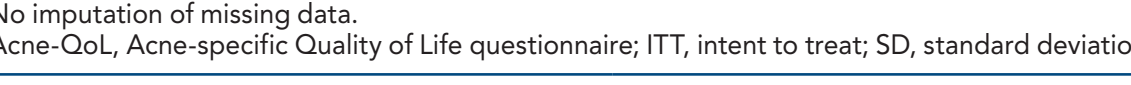

Safety and Tolerability

TEAE rates were higher for tazarotene-treated participants versus vehicle, though
most were unrelated to treatment (any TEAE: $31.2 \%$ vs $18.8 \%$; related

- The rates were similar to those seen in the overall safety population (n=779
tazarotenen, $=791$ vehicle) (any TEAE: $26.8 \%$ vs $19.1 \%$; related TEAE: 11.3\% vs 1.1\%) - For cutaneous safety and tolerability assessments, there were slight, transient
increaseses in scailing, itching, burning, and stinging beginning at week 2 , though mean

- Only erythema was reported in more than $20 \%$ of participants in either treatment arm
at baseline; as such, only these data are shown here (Figure 4 ) - In tazarotone-treated participants, reports of erythema decreased by week 12 and
mean scores remained at or below 0.5 for all weeks
FIGURE 4. Investigator-Assessed Erythema by Visit in White Adults

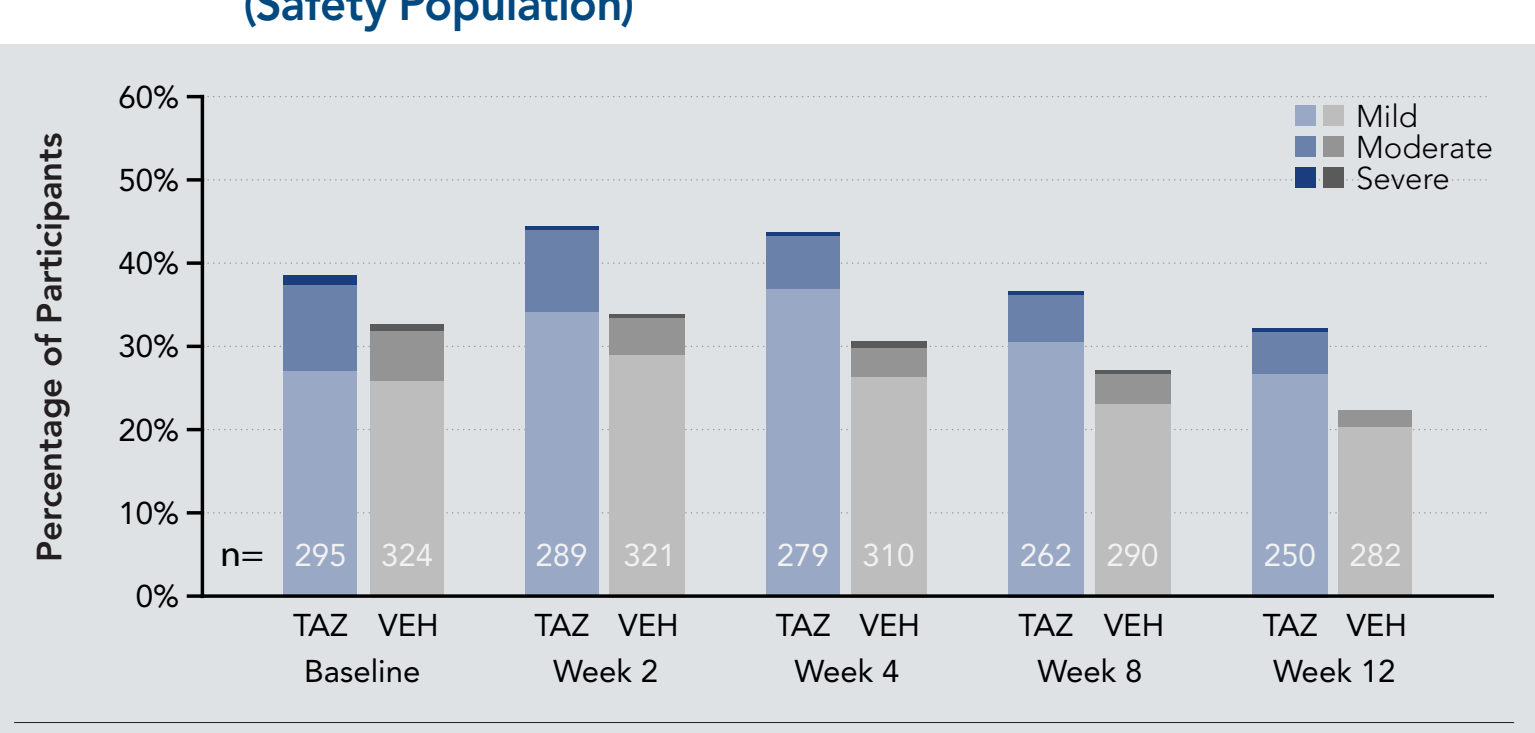

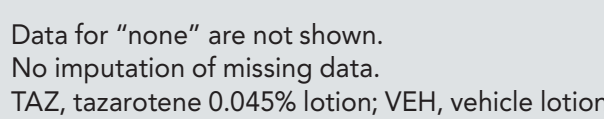

CONCLUSIONS

- Tazarotene $0.045 \%$ lotion was efficacious and well tolerated over 12 weeks, and led to quality-of-life improvements in White adults with moderate-to-severe acne; results were similar to the

overall population'

- Three-quarters of tazarotene-treated participants achieved mild, almost clear, or clear skin, with fewer participants beling moderate-severe withema par

- The hydrating and moisturizing polymeric emulsion han half the concentration of tazarotene versus other commercially available $0.1 \%$ formulations; this may help minimize instances of retinoid-induced erythema in White participants

- These results, combined with those from patients with skin of color, show that once dalily tazarotene $0.045 \%$ lotion is an effective and well tolerated treatment option regardless of skin

REFERENCES

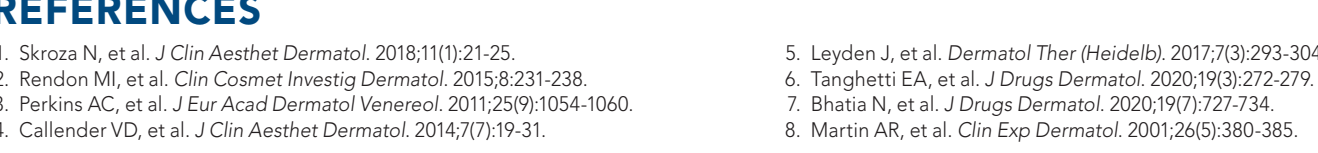

AUTHOR DISCLOSURES

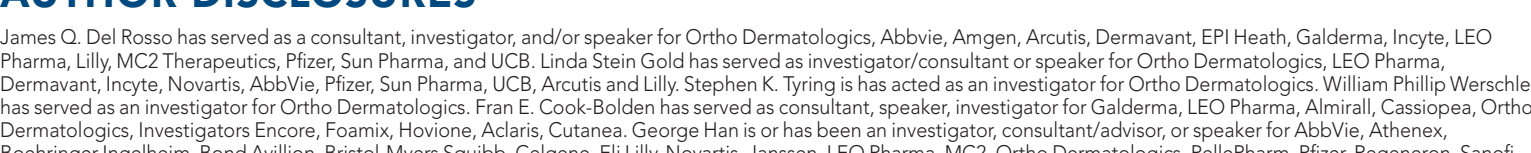

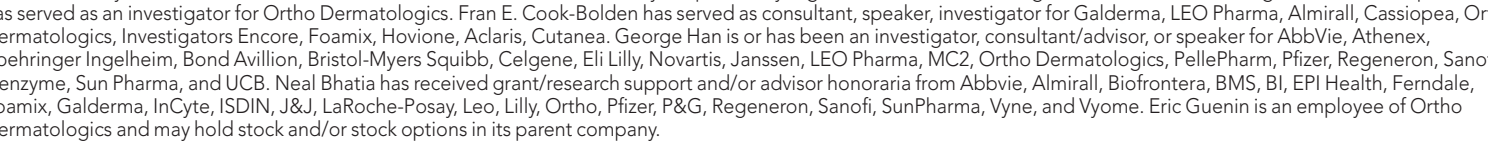

